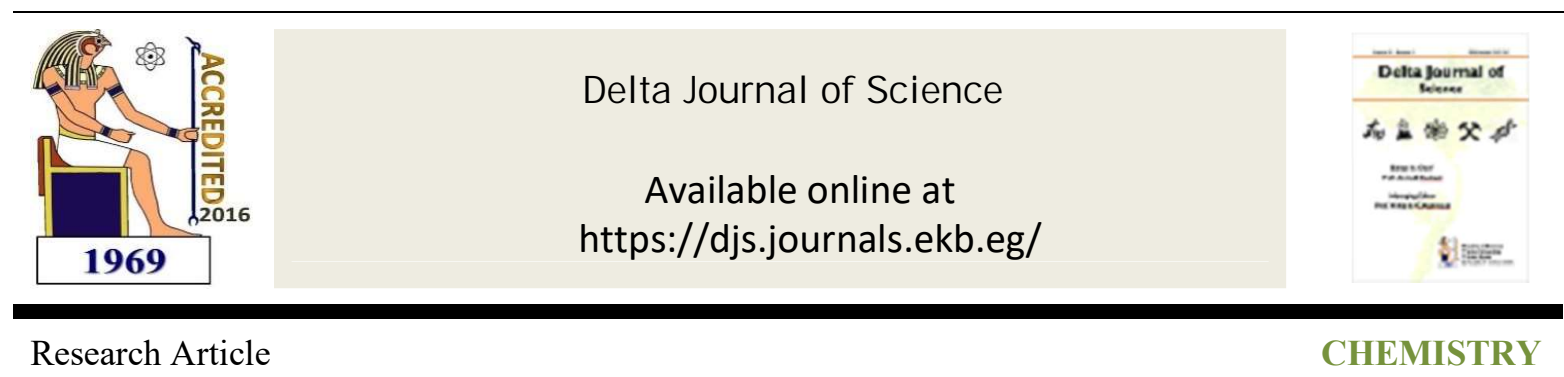

\title{
Synthesis and characterization of modified Poly(Ethylene-Co-Vinyl Alcohol) and its application as a biocidal agent
}

\author{
El-Refaie S. Kenawy, Mohamed M. Azaam *, Mohamed A Bishr \\ Polymer Research Group, Department of Chemistry, Faculty of Science, Tanta University, Tanta, 31527 Egypt \\ * Correspondence: Mohamed M. Azaam, Tel.: +201227171142 \\ E-mail:mmkazaam@yahoo.com,Mohmed.azam@science.tanta.edu.eg
}

KEY WORDS

$\mathrm{P}(\mathrm{EVOH})$, Biocidal,

Chloroacetylation,

Antimicrobial polymers
ABSTRACT

Co-polymeric materials of Poly(Ethylene-Co-Vinyl Alcohol) with different ratios, $\mathrm{P}(\mathrm{EVOH})$, were chemically modified through a series of reactions including chloroacetylation followed by the amination reaction. The acetyl, amine and sulfide groups were estimated through the Fourier Transform Infra-Red Spectra (FTIR) and the Elemental analysis. The antimicrobial activity of the modified copolymers was tested against various species by the cut plug procedure. It was concluded that the modified copolymers exhibited a satisfied antimicrobial activity towards both Gram positive and Gram negative bacteria. The highly observable result was estimated towards the protous species, at a ratio of $27 \%$ Ethylene and $73 \%$ Vinyl Alcohol since the inhibition zone reached $41 \mathrm{~mm}$. The antimicrobial activity increased by increasing the percentage of ethyl groups in the copolymeric material.So, the synthesized polymers were used as biocidal agents for water treatment.

\section{Introduction}

The microbiological contamination of water causes various negative effects on the human health, because of the spread of infections, and the quality control difficulties of the microbial corrosion and fouling, caused by the biofilm layer [1]. The conventional methods for water treatment concludes different mechanical and physical process [ $[\underline{3}, \underline{3}]$.
Furthermore, the chemical treatments have been applied, such as the application of different chemical agents (disinfectants, biocides) to inactivate microorganisms[ $[\underline{4}$, 5] .Chlorination is the most applied treatment that involves some difficulties, as the evaluation of toxic byproducts[6]. The infection by pathogenic microorganisms has a 
great potential in various applications, particularly in the medical fields, health care, and hygienic applications, textiles, water treatment, dental surgery and food industries [7],[]. Antimicrobial agents are defined as materials that are able to kill pathogenic microorganisms [8]. The application of antimicrobial copolymers decreases the environmental impact related to the conventional antimicrobial agents by the reduction of their residual toxicity, and hence optimization of their efficacy through controlling their lifetime. Moreover, the antimicrobial polymers are nonvolatile chemically stable agents that cannot invade through the skin. In this area, infections related to the biomaterials, signify a certain task to the medical implants [9-11]. Efficient copolymers is referred to both the functional groups and to their polymeric nature that their features be influenced by the extraordinarily size of the polymeric molecules [12-14]. Antimicrobial mediators are applied for various applications, for instance water disinfection, medications, sterilization, and the inhibition of the microbial growth[13],[15-17]. Ethylene vinyl alcohol copolymer (EVOH) is a semi-crystalline copolymer of ethylene and vinyl alcohol monomeric subunits. $\mathrm{P}(\mathrm{EVOH})$ is a well-known flexible thermoplastic copolymer for packaging, funnel tanks, and different medical techniques [18]. The synthesis of EVOH has been adjusted depending on their applications, for example, films, nanoparticles, and nanofibers[19, 20]. Several reasons for the selection of $\mathrm{EVOH}$ in this research. First, the polymeric material is highly hydrophilic because of the vinyl alcohol subunits. Second, it is a waterinsoluble referred to the ethylene subunits. Third, the good biocompatibility of EVOH; and finally, the other hydroxyl functionality exhibits chances for chemical modification[2, 21, 22]. Actually, several literatures have demonstrated the application of EVOH as a biomaterial [23]. Juliana and Maria [24]reported that $\mathrm{P}(\mathrm{EVOH})$ with different ethylene percentage and poly(methyl methacrylate) (PMMA) were synthesized by the mechanical grinding in the melted phase.
Roberto et al [25] demonstrated the singlescrew extrusion procedure to obtain composite films of $\mathrm{P}(\mathrm{EVOH})$. The recent research aimed to investigate the synthesis of a novel antimicrobial copolymers of $\mathrm{P}(\mathrm{EVOH})$ for water treatment.

\section{Experimental}

\section{Materials and Methods}

Poly(ethylene-co-vinyl alcohol), purity 99\% was purchased from Sigma Aldrich, Germany. Chloroacetyl chloride and Glacial acetic acid were purchased from Elgoumhouria Co. (Cairo, Egypt). Pyridine was purchased from El-Nasr Pharmaceutical Chemicals (Egypt). Ethylene diamine (EDA) and Propylene diamine (PDA)were purchased from Acros (New Jersey, USA).

\section{Test microorganisms and media}

The tested microorganisms were Escherichiacoli, Streptococcusaureus, Klepsiellasp and Proteus sp in addition to some fungi such as Asperigillusniger and pathogenic Candida spp. (C. krusei, C. albican, C. tropicalis and C. glabrata). The tested microorganism was obtained from the Bacteriology and Mycology unit, Botany Department, Faculty of Science, Tanta University.

\section{Chloroactylation of poly (ethylene co vinyl alcohol)}

Pyridine $(55 \mathrm{~mL})$ was added to a suspension of EVOH $(27 \%, 32 \%, 38 \%$ and $44 \%)$ $(10 \mathrm{~g})$ in $100 \mathrm{~mL}$ DMSO and the mixture was cooled to $0^{\circ} \mathrm{C}$. Chloroacetylchloride $(55$ $\mathrm{mL}$ ) was added dropwise and stirred at $0{ }^{\circ} \mathrm{C}$ for $3 \mathrm{~h}$ and room temperature for further 4 days .The insoluble part was removed by filtration and the filtrate was collected and concentrated on the rotary evaporator. The product was precipitated by adding distilled water, filtered off washed with water and was dried under vacuum at $40^{\circ} \mathrm{C}$ for two days. 


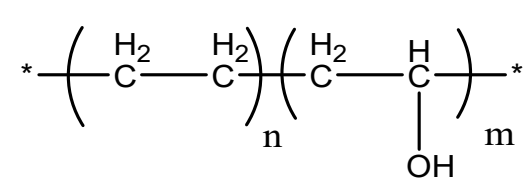

Ethylene-Vinyl Alcohol (EVOH)

Scheme1: Chloroactylation of poly(ethylene co vinyl alcohol)

\section{Amination of chloroacetylated \\ poly(ethylene-co-vinyl alcohol)}

\section{Amination with Ethylene diamine}

A suspension of copolymer (1) (3 g) is added to $30 \mathrm{~mL}$ of ethylene diamine. The reaction mixture was stirred at $90^{\circ} \mathrm{C}$ for $7 \mathrm{~h}$, then remain the reaction mixture to complete for $24 \mathrm{~h}$. The product was filtered off, washed with distilled water then with ethanol to remove the excess of ethylene diamine. The product was dried at $40{ }^{\circ} \mathrm{C}$ under vacuum for two days.

Table (1): Feed composition of the different prepared CRUF samples.

\begin{tabular}{ccc}
\hline Sample code & Urea Wt\% & $\begin{array}{c}\text { Composite } \\
\text { Wt\% }\end{array}$ \\
\hline CRUF1 & 90 & 10 \\
CRUF2 & 80 & 20 \\
CRUF3 & 70 & 30 \\
CRUF4 & 60 & 40 \\
CRUF5 & 50 & 50 \\
\hline
\end{tabular}

\section{Study of urea release from the CRUF matrix in water}

The release behavior of urea from the CRUF in distilled water was studied according to the following procedure: $0.3 \mathrm{~g}$ of the CRUF was contained in a tea bag-like tissue and then immersed in $200 \mathrm{ml}$ of distilled water in a glass beaker properly covered and kept at were the same as that of loaded CRUF) were also carried out, respectively.

\section{Results and discussions}

replenished. Detection of released urea was performed using UV-Vis spectrophotometer. According to Ehrlich reaction, $\mathrm{P}-\mathrm{N}, \mathrm{N}^{\prime}$ dimethylamino benzaldehyde reacts with urea to give a lemon Kelly color which would absorb visible light at $440 \mathrm{~nm}$. All the release experiments were done in a triplicate, and their results were averaged.

\section{Release of urea from CRUF in soil}

In order to demonstrate the release of urea from superabsorbent composite in agricultural field, release experiments in soil column were done. The soil used in this experiment was airdried and sieved to less than $2 \mathrm{~mm}$. Polyvinyl chloride (PVC) pipe of $45 \mathrm{~mm}$ internal diameter was used to hold soil. Loaded CRUF ( 2 g) was well mixed with $200 \mathrm{~g}$ dry sandy soil and placed in the PVC pipe. The bottom of the tube was sealed by nylon fabric. Water was added slowly by a perfusion tube (one drop every $10 \mathrm{~s}$ ). The time started when water seeped from the bottom of the pipe, i.e. water saturation. The leached water was collected, from which urea was estimated by the ultraviolet spectrophotometer [7]. Control experiments, i.e. urea, (the amounts of urea

2.1. Release behavior of urea from the controlled release fertilizer (CRUF)

The study of the release of urea from superabsorbent composite was carried out as described in the experimental part. The best 
composite-urea formulation that can give good controlled release behavior was selected. To investigate the properties of the selected composite as a new controlled release system, various formulations were prepared from the selected composite and urea as shown in table (1). The release measurements were carried out in distilled water at room temperature $\left(25^{\circ} \mathrm{C}\right)$ and the release was monitored using UV spectrophotometer at $440 \mathrm{~nm}$.

The studies were carried out for 16 hours the results showed clearly that on increasing the amount of composite in the formulation from $10 \mathrm{wt} \%$ to $50 \mathrm{wt} \%$, the release rate decrease and release time increased from 3 hours (in case of $\mathrm{CRUF}_{1}$ ) to 16 hours (in case of CRUF5).

Figure (1) depicts the release profiles of urea from loaded controlled release urea fertilizer (CRUF) with different urea loading percentage in distilled water (swollen in aqueous solutions of urea with different concentrations). Even though pure urea dissolve in water entirely in five seconds, CRUF $_{1}$ released $100 \%$ of its urea after 3 hours while the $\mathrm{CRUF}_{2}$ released $100 \%$ of urea after 6 hours. Similar results were obtained for $\mathrm{CRUF}_{3}, \mathrm{CRUF}_{4}$ and $\mathrm{CRUF}_{5}$ which released $100 \%$ of urea in 10,13 and 16 hours, respectively.

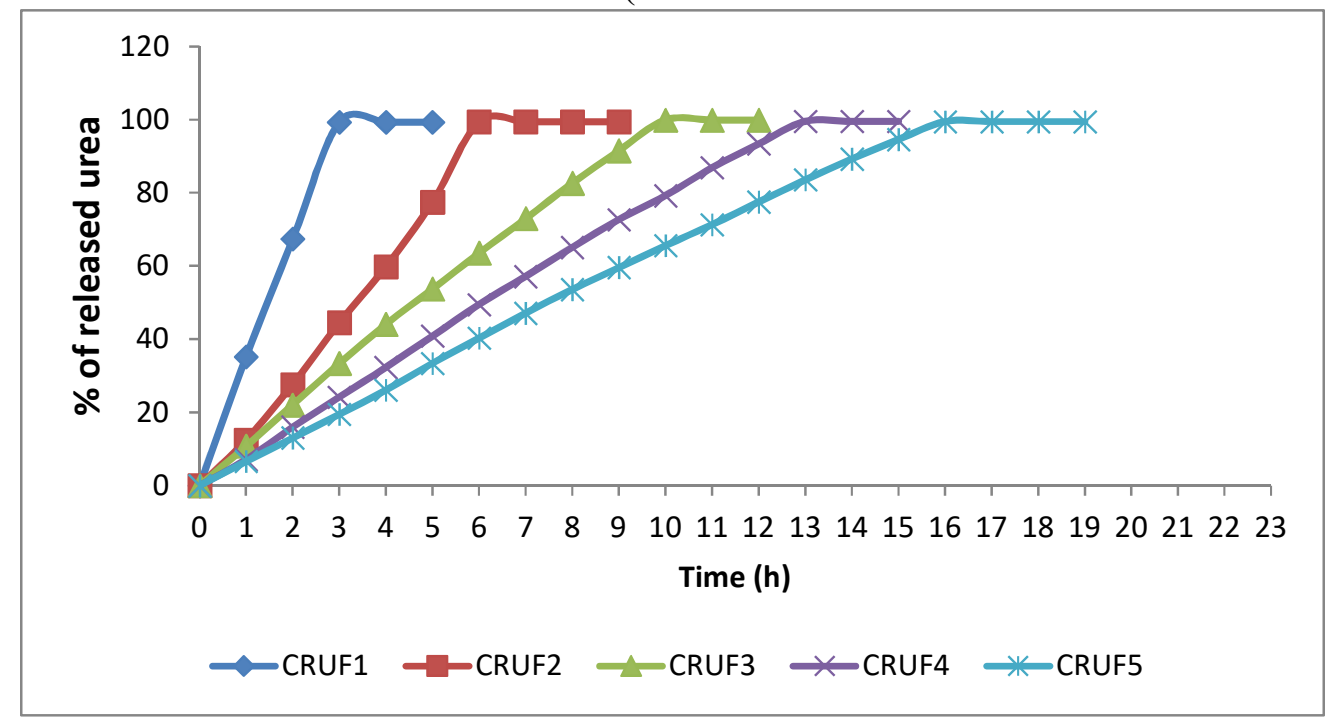

Fig. (1): The release rate of urea from different CRUF formulations in distilled water.

It was found that during the first hour of release study, the CRUF1 released $35 \%$ of urea, while $\mathrm{CRUF}_{2}$ released $12.5 \%$ of its urea content. The decrease of percentage of the released urea was repeated with the other formulations. The first hour release of $\mathrm{CRUF}_{3}$, $\mathrm{CRUF}_{4}$ and $\mathrm{CRUF}_{5}$ were $10.8 \%, 7 \%$ and $6.5 \%$ respectively. The high percent of the released urea from $\mathrm{CRUF}_{1}$ could be due to the release of urea adsorbed on the composite surface and pores formed on the surface CRUF.

The decrease in the rate of the released ureacomposite formulation was noted by increasing the superabsorbent composite content. This could be attributed to the fact that the release is diffusion controlled. Therefore, on increasing the percent of
The formed pores on the surface may have led to easy penetration of water vapor to the urea entrapped in the core causing the initial release of urea in the first hour of release study as the coating composite has not reached the swelling equilibrium yet. After the swelling equilibrium is reached, all the pores on the surface may have been closed forcing urea release from the CRUF to follow a diffusion mechanism from the interlayers of the composite.

superabsorbent composite, more barriers are formed which decreases the diffusion rate and consequently the release rate of urea. After the swelling equilibrium was achieved, the perfect swollen samples were formed. 


\section{The effect of $\mathrm{pH}$ on the release of urea from the controlled release fertilizer (CRUF5)}

To investigate the effect of the $\mathrm{pH}$ of the release media (aqueous media) on the release rate of urea, the release was conducted in the different $\mathrm{pHs}$ media ( $\mathrm{pH} 4,7$ and 10).

Figure (2) shows that there was a slight difference in the release behavior of urea from the $\mathrm{CRUF}_{5}$ in the various $\mathrm{pHs}$ that $100 \%$ released after 11,16 and $14 \mathrm{~h}$ for $\mathrm{pHs} 4,10$

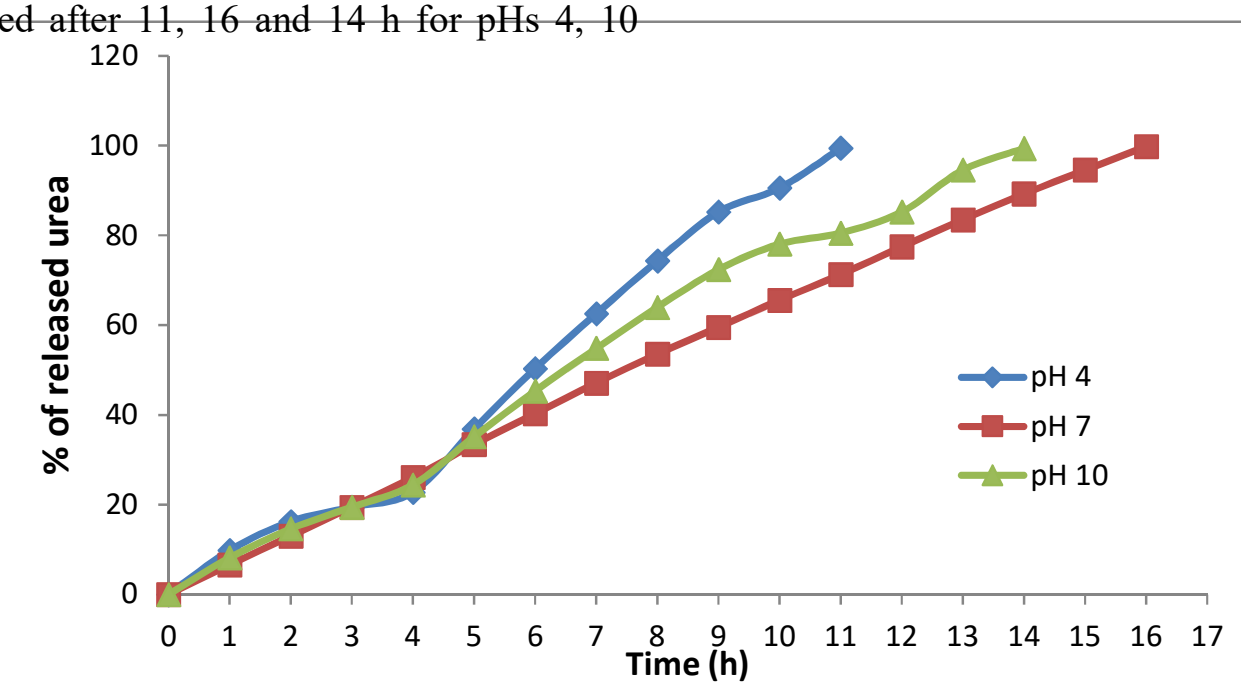

Fig. (2): The release rate of urea from CRUF5 in different media.

\section{Release of urea from CRUF in soil}

Because there are many factors that could affect the release of urea from loaded CRUF in soil, such as the kind of soil, cation exchange capacity (CEC), water content, bulk density, urea concentration, gel collapse and so on, there must exist obvious differences for urea release from loaded CRUF between in distilled water and in soil. Therefore, we measured the urea release behaviors in soil.

Figure (3) shows the urea release behaviors of loaded CRUF and untreated urea in soil. The urea release rate of untreated urea was very fast (as shown in figure (3). While the urea release rate of loaded CRUF decreased obviously compared with that of urea, the result was in agreement with that of Smith [8], 2) Urea could be released or desorbed slowly through the dynamic exchange of free water between soil solution and CRUF [9].

3) The released urea was purged out of the soil by water.

The reasons for the slower release rate in soil than in water may be due to the existing of many kinds of ions in soil solution, the and 7 respectively. This could be attributed to the presence of different ions. The presence of cations like $\mathrm{Na}^{+}, \mathrm{Ca}^{+2}, \mathrm{Mg}^{+2}, \mathrm{Fe}^{+3}, \mathrm{H}^{+}$and others lowered the swelling of the composite involved in CRUF5 formulation due to the screening effect of these cations on the anionic hydrophilic groups, which restricts the expanding of polymeric network and consequently increase the release rate of urea from the urea-composite formulation. 


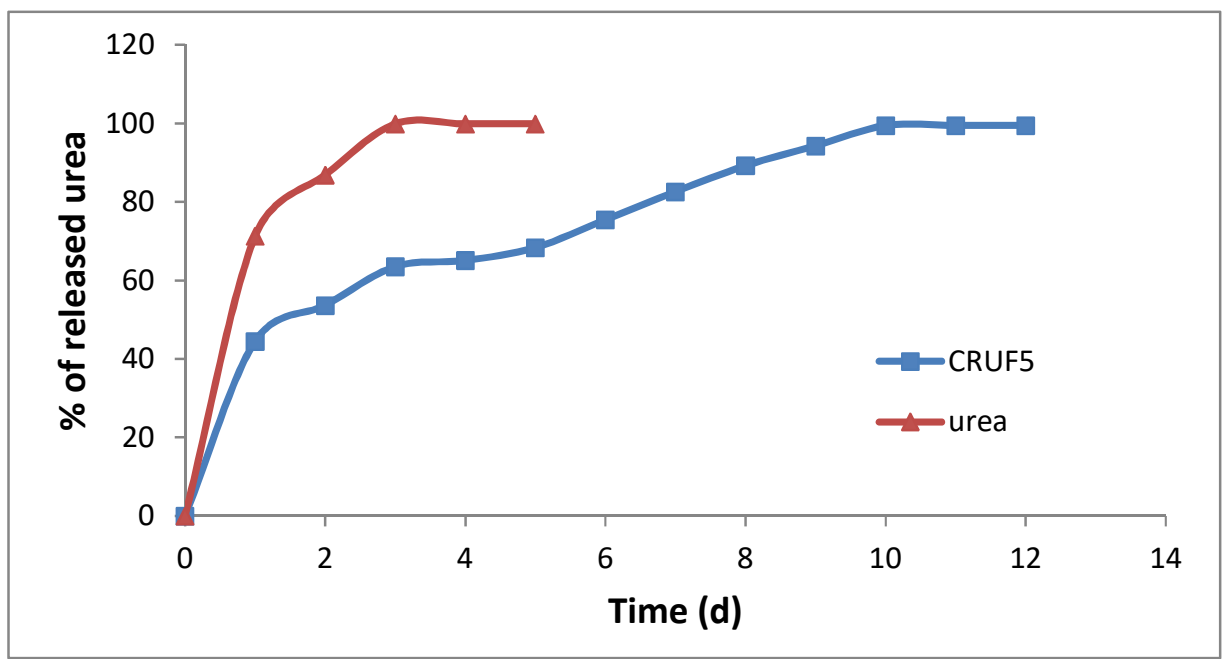

Fig. (3): The release rate of urea from CRUF5 in the soil.

\section{Conclusion}

The release study of urea from the formulated controlled release urea fertilizer (CRUF) showed that the rate of urea release from the formulated CRUF decreased with the increased composite content while $\mathrm{pH}$ had no evident effect on the release rate. The prepared CRUF showed that urea could last in soil for nearly 12 days while pure urea disappeared from soil after about 3 days only.

\section{Acknowledgment}

The authors extend their appreciation to the Science and Technology Development Fund (STDF) for funding this work through research project entitled "Superabsorbent polymer composite for agricultural applications" (Project ID: 5842).

\section{References}

[1] M. Trenkel, Slow- and Controlled-Release and Stabilized Fertilizers: An Option for Enhancing Nutrient Use Efficiency in Agriculture, Second edi., vol. 1. Paris, France: International Fertilizer Industry Association (IFA), 2010.

[2] T. Zheng, Y. Liang, S. Ye, and Z. He, "Superabsorbent hydrogels as carriers for the controlled-release of urea: Experiments and a mathematical model describing the release rate," Biosyst. Eng., vol. 102, no. 1, pp. 44-50, 2009.

[3] D. W. Rindt, G. M. Blouin, and J. G. Getsinger, "Sulfur coating on nitrogen fertilizer to reduce dissolution rate," J. Agric. Food Chem., vol. 16, no. 5, pp. 773-778, 1968.

[4] A. Shaviv, "Controlled release fertilizers," in IFA International Workshop on Enhanced-Efficiency Fertilizers, 2005.

[5] G. M. Blouin and D. W. Rindt, "Method of Making Sulfur-Coated Fertilizer Pellet Having a Controlled Dissolution Rate," 1967.
[6] O. E. M. Glenn M. Blouin, Donald W. Rindt, "Sulfur-coated fertilizers for controlled release. Pilotplant production," Agric. Food Chem., vol. 19, no. 5, pp. 801-808, 1971.

[7] G. W. Watt and J. D. Chrisp, "Spectrophotometric method for determination of urea," Anal. Chem., vol. 26, no. 3, pp. 452-453, 1954.

[8] J. D. Smith and H. C. Harrison, "Evaluation of polymers for controlled release properties when incorporated with nitrogen fertilizer solutions," Commun. Soil Sci. Plant Anal., vol. 22, no. 5-6, pp. 559-573, 1991.

[9] D. S. G. Hu and M. T. S. Lin, “Water-polymer interactions and critical phenomena of swelling in inhomogeneous poly(acrylonitrile-acrylamide-acrylic acid) gels," Polymer (Guildf)., vol. 35, no. 20, pp. 4416-4422, 1994.

[10] A. K. Bajpai and A. Giri, "Swelling dynamics of a macromolecular hydrophilic network and evaluation of its potential for controlled release of agrochemicals, " React. Funct. Polym., vol. 53, no. 2-3, pp. 125-141, 2002. 